\title{
Magnetic particles and strings in iron langasite
}

\author{
Evgenii Barts $\mathbb{( D}^{1 凶}$ and Maxim Mostovoy (iD)
}

Magnetic topological defects can store and carry information. Replacement of extended defects, such as domain walls and Skyrmion tubes, by compact magnetic particles that can propagate in all three spatial directions may open an extra dimension in the design of magnetic memory and data processing devices. We show that such objects can be found in iron langasite, which exhibits a hierarchy of non-collinear antiferromagnetic spin structures at very different length scales. We derive an effective model describing long-distance magnetic modulations in this chiral magnet and find unusual two- and three-dimensional topological defects. The order parameter space of our model is similar to that of superfluid ${ }^{3} \mathrm{He}-\mathrm{A}$, and the particle-like magnetic defect is closely related to the Shankar monopole and hedgehog soliton in the Skyrme model of baryons. Mobile magnetic particles stabilized in non-collinear antiferromagnets can play an important role in antiferromagnetic spintronics.

npj Quantum Materials (2021)6:104; https://doi.org/10.1038/s41535-021-00408-4

\section{INTRODUCTION}

The topology of defects in ordered states of matter is governed by the order parameter describing spontaneous symmetry breaking at a phase transition ${ }^{1}$. As the number of variables required to characterize an ordered state increases, so does the diversity and complexity of topological defects. A wide variety of defects is found in superfluid ${ }^{3} \mathrm{He}$ with the order parameter describing orbital momentum, spin, and phase of the condensate ${ }^{2,3}$.

Nontrivial topology does not necessarily make defects stable: a competition between interactions with different properties under the scaling transformation, $\mathbf{x} \rightarrow \Lambda \mathbf{x}$, is required to prevent the collapse of the defect ${ }^{4}$. Thus isolated Skyrmion tubes in chiral magnets with a diameter of $10-100 \mathrm{~nm}$ are stabilized by Dzyaloshinskii-Moriya (DM) interactions ${ }^{5,6}$ favoring non-collinear spins, which compete with the Zeeman and magnetic anisotropy energy favoring uniform states ${ }^{7-9}$. The small size and high stability of Skyrmion tubes in bulk chiral magnets and magnetic multilayers, as well as their dynamics, driven by applied electric currents, make them promising information carriers in magnetic memory and data processing devices ${ }^{10-12}$. Even smaller skyrmions have been recently observed in centrosymmetric magnets ${ }^{13-15}$, where they are stabilized by magnetic frustration and/ or long-ranged interactions between spins mediated by conduction electrons ${ }^{16-20}$.

Here, we discuss a realistic material that can host threedimensional (3D) magnetic Skyrmions-non-singular defects which, unlike the Skyrmion tubes, have a finite size in all three spatial directions. These magnetic particles can transfer information in all directions, stimulating the design of three-dimensional spintronic devices. 3D Skyrmions originally emerged as solitons in the non-linear meson model of T.H.R. Skyrme ${ }^{21}$. The parameter space of this model, formed by four meson fields, is three-sphere $S^{3}$ parametrized by three angles. A closely related defect, Shankar monopole, was predicted to exist in the A-phase of superfluid ${ }^{3} \mathrm{He}^{22,23}$. The order parameter describing this phase is an $\mathrm{SO}(3)$ matrix, and the collection of all possible ordered states is projective three-sphere $\mathrm{RP}^{3}$. Shankar monopole has been recently realized in the Bose-Einstein condensate of trapped spin-1 particles by application of time-dependent and spatially inhomogeneous magnetic fields ${ }^{24}$. This defect is, however, unstable and has a short lifetime.

Higher-dimensional order parameter spaces can also be realized in magnetic materials, in particular, antiferromagnets with triangle-based spin lattices showing a non-collinear $120^{\circ}$ ordering of spins in the triangles described by an $\mathrm{SO}(3)$ matrix ${ }^{25,26}$. Noncollinear antiferromagnetic (AFM) orders give rise to electron and magnon bands with non-trivial topology and Weyl fermions ${ }^{27-31}$ resulting in large anomalous Hall and Nernst effects ${ }^{32,33}$ that can be controlled electrically ${ }^{34}$.

We show that 3D skyrmions can naturally occur in the iron langasite, $\mathrm{Ba}_{3} \mathrm{TaFe}_{3} \mathrm{Si}_{2} \mathrm{O}_{14}$. This fascinating material is both magnetically frustrated and chiral. The Fe-langasite spin lattice is built of triangles formed by the $\mathrm{Fe}^{3+}$-ions in the $a b$ layers (see Fig. 1) with AFM Heisenberg exchange interactions between spins in the triangles resulting in a $120^{\circ}$ spin ordering ${ }^{35}$. Furthermore, competing exchange interactions between spins of neighboring triangles, stacked along the $c$-axis, give rise to a helical spiral modulation of the $120^{\circ}$-ordering with the period of $\sim 7$ lattice constants along the $c$-axis. The direction of the spin rotation in the spiral is governed by the chiral nature of the langasite crystal ${ }^{35-38}$ that, otherwise, has little effect on the spin structure. However, when the magnetic anisotropy is effectively reduced by an applied magnetic field, DM interactions give rise to an additional spiral modulation with a period of about $2000 \AA$ along a direction perpendicular to the $c$-axis ${ }^{39}$. We show that the same DM interactions can stabilize more complex modulated states as well as unusual topological magnetic defects, in particular, the magnetic particles carrying 3D Skyrmion topological charge and an associated Hopf number.

\section{RESULTS}

\section{Effective model}

The $120^{\circ}$ order of the classical unit spins $\mathbf{S}_{1}, \mathbf{S}_{2}, \mathbf{S}_{3}$ in triangles can be described by two orthogonal unit vectors, $\mathbf{V}_{1}$ and $\mathbf{V}_{2}{ }^{39,40}: \mathbf{S}_{1}=$ $\mathbf{V}_{1}, \mathbf{S}_{2}=-\frac{1}{2} \mathbf{V}_{1}+\frac{\sqrt{3}}{2} \mathbf{V}_{2}$ and $\mathbf{S}_{3}=-\frac{1}{2} \mathbf{V}_{1}-\frac{\sqrt{3}}{2} \mathbf{V}_{2}$, so that $\mathbf{S}_{1}+\mathbf{S}_{2}+$ $\mathbf{S}_{3}=0$. Spatial rotations of the frame formed by $\mathbf{V}_{1}, \mathbf{V}_{2}$ and $\mathbf{n}=$ $\mathbf{V}_{1} \times \mathbf{V}_{2}$ are described by $\mathrm{SO}(3)$ matrix $R$ parametrized by three

${ }^{1}$ Zernike Institute for Advanced Materials, University of Groningen, Nijenborgh 4, 9747 AG Groningen, The Netherlands. ${ }^{凶}$ email: e.barts@rug.nl 

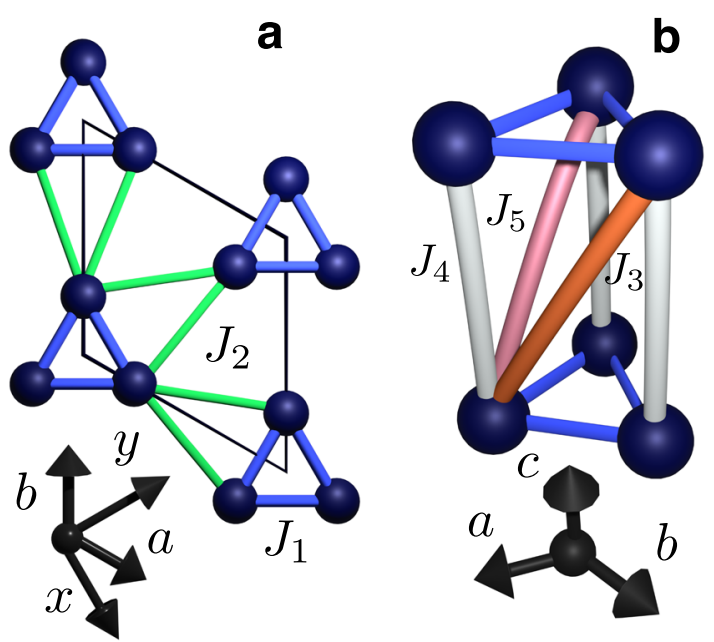

Fig. 1 The Fe-langasite crystal structure. a Spin triangles in the crystallographic $a b$ plane formed by $\mathrm{Fe}^{3+}$ ions (blue spheres). Blue lines mark the bonds between the spins in the triangles with the relatively strong AFM exchange interaction $J_{1}$. The triangles form a hexagonal lattice with the AFM coupling $J_{2}$ between the triangles. b Stacking of the spin triangles along the $c$ axis with the competing AFM interactions $J_{3}, J_{4}$, and $J_{5}$ between the spins in neighboring triangles.

Euler angles, $\phi, \theta$, and $\psi^{41}$ :

$\mathbf{V}_{1,2}=R \mathbf{V}_{1,2}^{(0)}=R_{z}(\phi) R_{y}(\theta) R_{z}(\Psi) \mathbf{V}_{1,2}^{(0)}$,

where $R_{z}$ and $R_{y}$ are the matrices of rotations around the $z$ and $y$ axes, respectively, $\theta$ and $\phi$ are the polar and azimuthal angles of the unit vector $\mathbf{n}=(\sin \theta \cos \phi, \sin \theta \sin \phi, \cos \theta)^{T}$ describing the direction of the vector chirality of the $120^{\circ}$ spin order (see Fig. 2), $\mathbf{V}_{1}^{(0)}=\hat{\mathbf{x}}$ and $\mathbf{V}_{2}^{(0)}=\hat{\mathbf{y}}$.

The short-period spiral ordering observed in Fe-langasite in zero magnetic field originates from the competing exchange interactions between the spin triangles stacked along the $c$ direction (see Fig. 1b). Importantly, the isotropic Heisenberg exchange interactions determine the spiral wave vector $Q \| c^{36}: \tan Q c=$ $\sqrt{3} \frac{\left(J_{5}-J_{3}\right)}{\left(2 J_{4}-J_{3}-J_{5}\right)}$, but not the orientation of the spiral plane described by the vector chirality $\mathbf{n}$. The latter is governed by DM interactions between spins in the triangles,

$D_{z}\left(\mathbf{S}_{1} \times \mathbf{S}_{2}+\mathbf{S}_{2} \times \mathbf{S}_{3}+\mathbf{S}_{3} \times \mathbf{S}_{1}\right)_{z}=\frac{3 \sqrt{3}}{2} D_{z} n_{z}$,

favoring a helical spiral with the helicity $\operatorname{sign}\left(n_{z} Q\right)^{35}$ and by an easy-plane magnetic anisotropy, which are two orders of magnitude weaker than the exchange interaction in the triangles $37,38,42$. On the other hand, the inter-triangle DM interactions in this chiral magnet tend to induce 'slow' variations of $\mathbf{n}$ and $\Psi$ giving rise to a long-period magnetic superstructure observed under an applied magnetic field ${ }^{39}$. The competition between the magnetic anisotropy favoring a unique direction of $\mathbf{n}$ and the tendency to large-scale modulations, both being relatively weak relativistic effects, can also stabilize topological magnetic defects that are superimposed on the fast spin rotations with the propagation vector along the $c$ direction.

To obtain an effective model describing long-period magnetic superstructures in Fe-langasite, we separate fast and slow variations of the order parameter by introducing a slowly varying angle $\psi(\mathbf{r}): \psi(\mathbf{r})=Q z+\psi(\mathbf{r})$. The energy is then expanded in powers of gradients of the three slowly varying angles $\theta, \phi$, and $\psi$, and averaged over the fast spin rotations (technical details of the derivation can be found in Supplementary Note 1). The energy

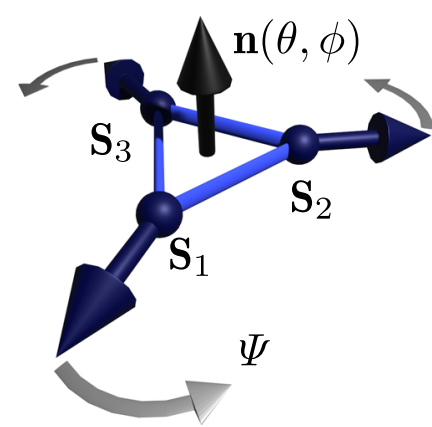

Fig. 2 The $120^{\circ}$ spin order. The order parameter is described by the polar and azimuthal angles, $\theta$ and $\phi$, of the vector chirality $\mathbf{n}=\frac{2}{3 \sqrt{3}}\left(\mathbf{S}_{1} \times \mathbf{S}_{2}+\mathbf{S}_{2} \times \mathbf{S}_{3}+\mathbf{S}_{3} \times \mathbf{S}_{1}\right)$, and the angle $\Psi$ of the spin rotation around $\mathbf{n}$.

density of the effective model is

$$
\begin{aligned}
\mathcal{E}= & \frac{J_{z}}{2}\left[\left(\partial_{z} \mathbf{n}\right)^{2}+2\left(D_{z} \psi\right)^{2}\right]+\frac{J_{\perp}}{2} \sum_{\mu=x, y}\left[\left(\partial_{\mu} \mathbf{n}\right)^{2}+2\left(D_{\mu} \psi\right)^{2}\right] \\
& +K_{1}(1-\cos \theta)+\frac{K_{2}}{2}\left(1-\cos ^{2} \theta\right)-\frac{x}{2}(\mathbf{H} \cdot \mathbf{n})^{2} \\
& +\lambda\left[\cos ^{2} \theta\left(-\sin \phi \partial_{x} \theta+\cos \phi \partial_{y} \theta\right)+\left(\mathbf{n} \cdot \partial_{\perp}\right) \psi\right],
\end{aligned}
$$

where the first term originates from the interlayer Heisenberg exchange interactions (see Fig. 1b) and $J_{z}=\frac{3}{4} \sqrt{\left(2 J_{4}-J_{3}-J_{5}\right)^{2}+3\left(J_{3}-J_{5}\right)^{2}}$. The second term with $J_{\perp}=$ $\frac{\sqrt{3}}{2} J_{2}$ results from the exchange interactions between the $\mathrm{Fe}$ triangles in the $a b$ layers (Fig. 1a). The distances in the direction parallel(perpendicular) to the $c$-axis of the hexagonal lattice are measured in units of the lattice constant, $c(a) . D_{i} \psi=\partial_{i} \psi+$ $\cos \theta \partial_{i} \phi$ is the covariant derivative of $\psi(i=x, y, z)$ invariant under an arbitrary global rotation of spins. The third term in Eq. (3), playing the role of an internal magnetic field, originates from DM interactions between spins in the triangles [see Eq. (2)] and the fourth term is the magnetocrystalline anisotropy. The next term is the coupling of the spiral ordering to an applied magnetic field $\mathbf{H}$, which favors $\mathbf{n} \| \mathbf{H}(x>0)$ since the magnetic susceptibility is the largest for spins rotating in the plane perpendicular to the field vector. The last term in Eq. (3) is a Lifshitz invariant (LI) $)^{6,7}$ allowed by the chiral nature of the langasite crystal, $\partial_{\perp}$ being gradient along with the in-plane directions (the derivation of LIs for this non-collinear antiferromagnet is discussed in the "Methods" section).

\section{Phase diagram}

In zero field, the anisotropy terms with $K_{1}, K_{2}>0$ confine spins to the $a b$ plane and stabilize the spiral state called uniform (U), as in this state $n_{z}=+1$ and $\psi=$ const. In enantiopure samples of Felangasite studied in experiments $n_{z}=-1^{35-37}$. The sign of $n_{z}$ does not affect the phase diagram. An applied magnetic field $H \perp c$ tends to re-orient the spiral plane, eventually turning it perpendicular to the field $(n \| H)$. The re-orientation of $\mathbf{n}$, which resembles the spin-flop transition in collinear antiferromagnets, activates LI that can stabilize two very different states with additional large-scale modulations.

Assuming that $\mathbf{n}$ and $\psi$ in the modulated states vary along a vector $\boldsymbol{\xi}=\left(\cos \phi_{\xi}, \sin \phi_{\xi}, 0\right)$ in the $a b$ plane (this assumption is verified by numerical simulations), we exclude $\psi$ from Eq. (3) using $\partial_{\xi} \psi=-\cos \theta \partial_{\xi} \phi-\frac{\lambda}{2\lrcorner_{\perp}}(\xi \cdot \mathbf{n})$ and obtain the energy that only depends on $\mathbf{n}$,

$$
\begin{aligned}
\mathcal{E} & =\frac{J_{\perp}}{2}\left(\partial_{\xi} \mathbf{n}\right)^{2}+K_{1}(1-\cos \theta)+\frac{K_{2}}{2}\left(1-\cos ^{2} \theta\right) \\
& -\frac{\chi H^{2}}{2} n_{x}^{2}-\frac{\lambda^{2}}{4 J_{\perp}}(\xi \cdot \mathbf{n})^{2}-\lambda \sin ^{2} \theta \sin \left(\phi-\phi_{\xi}\right) \partial_{\xi} \theta,
\end{aligned}
$$

for $H \| x$ (see Fig. 1a). 
Figure 3 shows the phase diagram in the $\left(K_{1}, H\right)$ plane. In contrast to collinear antiferromagnets, the order parameter $\mathbf{n}$ does not abruptly flop but rotates continuously away from the $z$-axis in the $x z$ plane. For $H>H_{R}: X H_{R}^{2}=\left|K_{1}\right|+K_{2}-\frac{\lambda^{2}}{2 J^{\prime}}$, the rotation angle is given by

$$
\cos \theta=\frac{K_{1}}{x H^{2}+\frac{\lambda^{2}}{2 J_{\perp}}-K_{2}} .
$$

While $\mathbf{n}$ is constant, the angle $\psi$ varies monotonically, $\psi=q(\mathbf{r} \cdot \xi)$ with $q=-\frac{\lambda}{2} \sin \theta$, corresponding to an additional rotation of spins around $\mathbf{n}$ (see Fig. 4b) recently observed in Fe-langasite above $H_{R} \sim 4 T^{39}$. The wave vector $q$ increases as the field strength grows and $\mathbf{n}$ approaches the field direction. This 'tilted spiral' (TS) state with $\mathbf{n}$ tilted away from the c-axis has both helical and cycloidal components.

In another kind of modulated state, the domain wall array (DWA) shown in Fig. $4 \mathrm{a}, \psi$ is constant whereas $\mathbf{n}$ rotates in the $x z$ plane along the $y$-axis perpendicular to the applied field $\left(\phi-\phi_{\xi}=\frac{\pi}{2}\right)$. This state only appears for relatively small $K_{1}$ (see Fig. 3). At a critical field, $H_{\mathrm{c} 1}$, the energy of the domain wall, across which $\theta$ varies by $2 \pi$, vanishes, which marks the transition from the uniform spiral state to the DWA state. As $H$ increases further, the domain wall energy becomes negative, and the domain walls

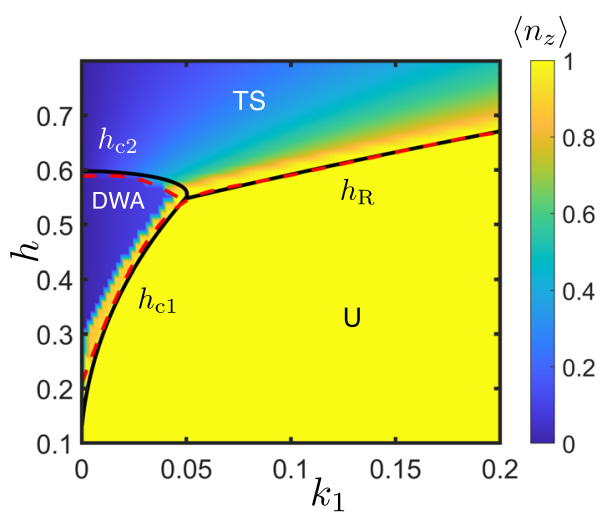

Fig. 3 Magnetic phase diagram of the model. The competing phases are: the 'uniform' (U) spiral state with $n \| z$ and $\psi=0$, the modulated 'tilted spiral' (TS) state and the domain wall array (DWA). Solid black(dashed red) phase transition lines are obtained analytically(numerically), the difference being a finite-size effect in numerical calculations. Color indicates the average $n_{z}$. $k_{1,2}=K_{1,2} /\left(\frac{\lambda^{2}}{2}\right)$ and the dimensionless magnetic field, $h$, is defined by $X H^{2}=h^{2} \frac{\lambda^{2}}{2}$. This calculation was performed for $k_{2}=1.25, \lambda=0.4$ and $J=1.0$. form an array with the period that decreases with the field. This state is similar to the 'mixed state' in collinear antiferromagnets ${ }^{43}$, except that in our case $\mathbf{n}$ rotates through the angle $2 \pi$ across the wall, since the uniform states with $n_{z}= \pm 1$ have different energies for $K_{1} \neq 0$. At the second critical field, $H_{\mathrm{c} 2}$, the transition between the DWA and TS states occurs and the modulation direction described by $\xi$ rotates abruptly through $90^{\circ}$.

Although the energy of all states in the phase diagram Fig. 3 can be found analytically (see Supplementary Note 3), we also performed numerical simulations of the model Eq. (3) rewritten in terms of two orthogonal unit vectors, $\mathbf{V}_{1}$ and $\mathbf{V}_{2}$ (see the "Methods" section), which confirm the phase diagram Fig. 3. We also found metastable multiply periodic states: the vortex array with a square lattice (Fig. $5 a, b$ ), the vortex chains (Fig. 5c, d) and the hexagonal crystal of coreless vortices (Fig. 5e, f), which can be stabilized by thermal fluctuations at elevated temperatures.

\section{Topological magnetic defects in two spatial dimensions}

Singular topological defects in a model with an $\mathrm{SO}(3)$ order parameter in two spatial dimensions $-Z_{2}$ vortices with energy logarithmically diverging with the system size-have been discussed in ref. ${ }^{25}$. Here we study non-singular finite-energy defects in the uniform ground state. One might think that, similarly to magnetic Skyrmions, such defects can be classified by the topology of $\mathbf{n}(x, y)$-textures after the angle $\psi$ is integrated out from Eq. (3), as it was done for one-dimensional states. However, in two spatial dimensions, the resulting energy functional, $E[\mathbf{n}]$, contains long-ranged Coulomb interactions between the 'electric' charges induced by spatial variations of $\mathbf{n}$. These interactions suppress Skyrmions, which are 'charged' and have an infinite 'electrostatic' energy.

The electrostatic potential, $\varphi_{\mathrm{el}}$, is a variable dual to $\psi$

$D_{\mu} \psi+\frac{\lambda}{2 J_{\perp}} n_{\mu}=-\epsilon_{\mu \nu} \partial_{v} \varphi_{\mathrm{el}}$,

where $\epsilon_{\mu v}$ is the antisymmetric tensor $(\mu, v=x, y)$. The divergence of the left-hand side is 0 as a result of the global gauge invariance: Eq. (3) is unchanged under $\psi \rightarrow \psi+a$. The electrostatic potential satisfies Poisson equation, $-\Delta \varphi_{\mathrm{el}}=4 \pi \rho_{\mathrm{el}}$, with the electric charge density, $\rho_{\mathrm{el}}=\frac{1}{4 \pi}\left(\mathbf{n} \cdot \partial_{x} \mathbf{n} \times \partial_{y} \mathbf{n}\right)-\frac{\lambda}{8 \pi J_{\perp}}[\nabla \times \mathbf{n}]_{z}$, the first term being the Skyrmion charge density. Equation (3) can then be written in the form

$\mathcal{E}=\frac{J_{\perp}}{2} \sum_{\mu=x, y}\left(\partial_{\mu} \mathbf{n}\right)^{2}-\lambda n_{z}[\nabla \times \mathbf{n}]_{z}+U(\mathbf{n})+\frac{1}{2 \epsilon} \varphi_{\mathrm{el}} \rho_{\mathrm{el}}$,

where $U(\mathbf{n})=K_{1}(1-\cos \theta)+\frac{\left(K_{2}-\frac{\lambda^{2}}{2 \perp_{\perp}}\right)}{2} \sin ^{2} \theta-\frac{X}{2}(\mathbf{H} \cdot \mathbf{n})^{2}$ and the last term is the positive electrostatic energy with the 'dielectric'
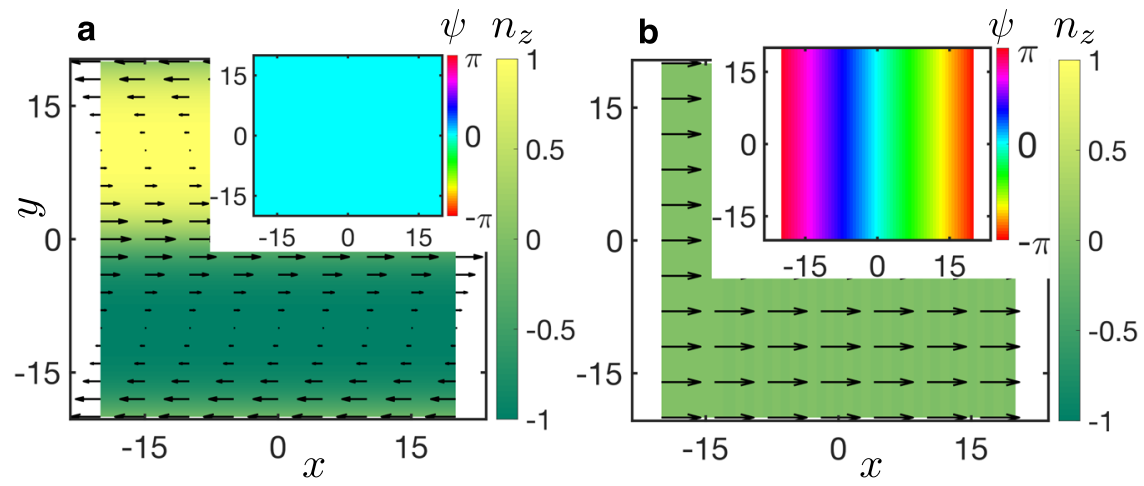

Fig. 4 Modulated states induced by $\boldsymbol{H} \| \boldsymbol{x}$. a The domain wall array, in which the angle $\theta$ rotates in the $x z$ plane along the $y$-axis normal to the field vector. $\mathbf{b}$ The 'tilted spiral' state with $n \| H$ and $\psi$ varying along the field direction. The figures show the vector chirality $\mathbf{n}$ and the corresponding angle $\psi$ is shown in the insets. In-plane components of $\mathbf{n}$ are indicated with arrows; $n_{z}$ and $\psi$ are color-coded. 

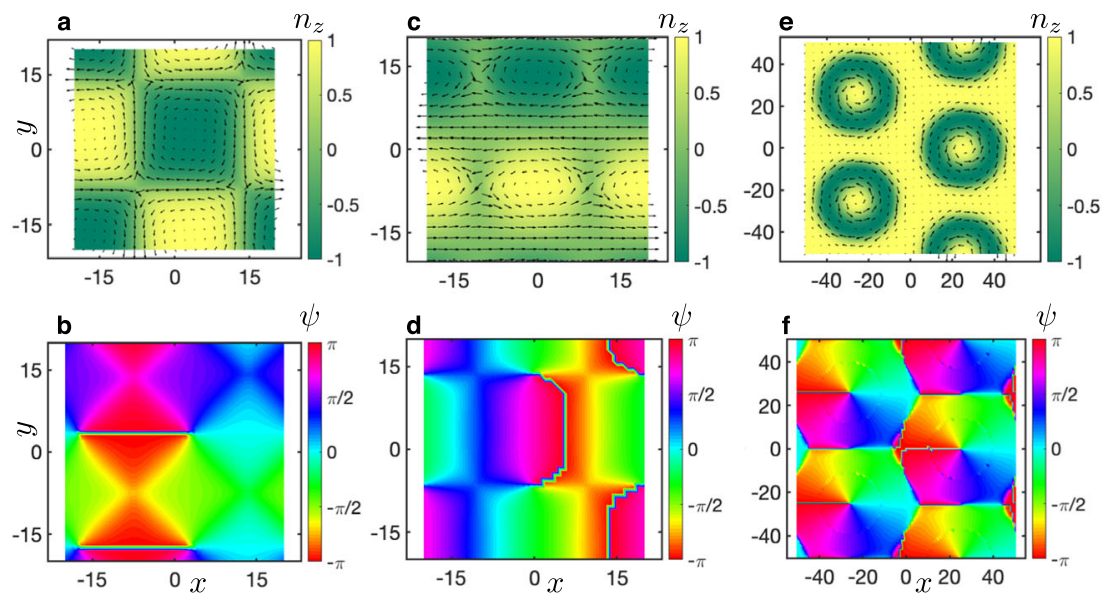

Fig. 5 Metastable modulated states. a, b The vortex array with a square lattice found at low applied magnetic fields $\left(H<H_{\mathrm{R}}\right)$. $\mathbf{c}$, $\mathbf{d}$ The alternating strings of merons and antimerons found at large applied magnetic fields $\left(H>H_{\mathrm{R}}\right)$. e, $\mathbf{f}$ The non-singular hexagonal vortex crystal. The first row (panels $\mathbf{a}, \mathbf{c}$ and $\mathbf{e}$ ) shows the vector chirality $\mathbf{n}$ and the second row (panels $\mathbf{b}, \mathbf{d}$ and $\mathbf{f}$ ) shows the corresponding angle $\psi$. In-plane components of $\mathbf{n}$ are indicated with arrows; $n_{z}$ and $\psi$ are color-coded. The angle $\psi$ is plotted modulo $2 \pi$ and the lines in the $\psi$-plots are branch cuts, across which $\psi$ discontinuously changes by $2 \pi$.
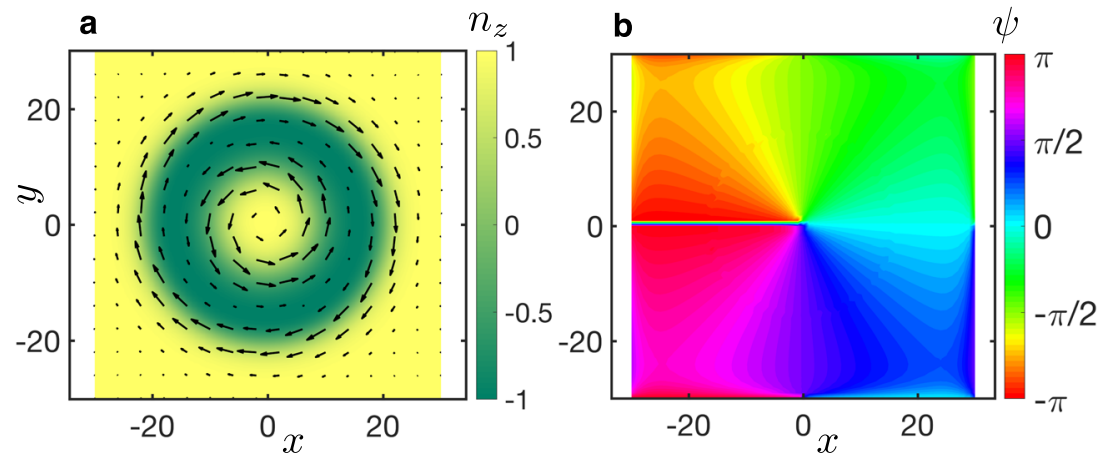

Fig. 6 Coreless vortex in the uniform spiral state. This topological defect consists of a target skyrmion formed by the vector chirality $\mathbf{n}$ (panel b) and a vortex, in which the angle $\psi$ varies by $-2 \pi$ along a closed loop around the center of the defect (panel b). Arrows show in-plane components of $\mathbf{n} ; n_{z}$ and $\psi$ are color-coded. The numerical simulation was done for $J_{\perp}=1, \lambda=0.34, K_{1}=0, K_{2}=0.1$, and $\mathbf{H}=0$.

constant $\epsilon=\frac{1}{8 \pi J}$. Finite-energy defects have zero total electric charge,

$Q_{\mathrm{el}}=\int d^{2} x \rho_{\mathrm{el}}=Q_{\mathrm{sk}}-\frac{\lambda}{8 \pi J_{\perp}} \oint d \mathbf{x} \cdot \mathbf{n}=0$,

where $Q_{s k}$ is the Skyrmion charge (Eq. (8) is similar to the Mermin-Ho relation for the circulation of the superfluid velocity in ${ }^{3} \mathrm{He}-\mathrm{A}^{44}$. Since for a finite-energy defect the integral over the infinite-radius circle in Eq. (8) is $0, Q_{s k}=0$, in agreement with $\pi_{2}(\mathrm{SO}(3))=0$.

A stable finite-energy defect in the spiral state with $n \| z$ is shown in Fig. 6 . In polar coordinates $(\rho, \varphi), \phi=\varphi+\frac{\pi}{2}, \psi=-\varphi$ and $\theta=\theta(\rho)$ monotonically increases from 0 at $\rho=0$ to $2 \pi$ at $\rho=\infty$. The $\mathbf{n}$-configuration shown in Fig. 6) $\mathbf{a}$ is that of a target skyrmion ${ }^{45-47}$ with $Q_{\text {sk }}=0$ and the angle $\psi$ forms a vortex with the winding number -1 (Fig. 6b). As in the vortices in type-II superconductors, the covariant derivative $D_{\mu} \psi$ vanishes far away from the vortex. However, it also vanishes at $\rho=0$, so that the $\psi$ vortex has no core. Note that $\phi+\psi=$ const in the vortex center, where $\theta=0$, corresponds to non-rotating spins.

This defect is stabilized by the LI in Eq. (3), which favors $\psi$ varying along $\mathbf{n}$ and $\theta$ varying along the direction normal to $\mathbf{n}$. Both these trends are fulfilled in the coreless vortex. Topological protection is ensured by the existence of a non-contractible loop in the $S O(3)$ manifold: $\pi_{1}(S O(3))=Z_{2}$. A path from the center of the defect to infinity along any radial direction is such a loop. In the center of the defect and at spatial infinity $n_{z}=+1$, whereas inside the green ring in Fig. $6 a n_{z}$ is negative, corresponding to the reversal of both the vector chirality of spins in triangles and the spiral helicity. The rotational symmetry of the defect turns the calculation of $\theta(\rho)$ into a one-dimensional problem (see Supplementary Note 4).

\section{D Skyrmion}

The third homotopy group, $\pi_{3}(\mathrm{SO}(3))=Z$, allows for particle-like topological defects that have a finite spatial extent in all three directions. They are closely related to hedgehog solitons in the nonlinear meson model of T.H.R. Skyrme ${ }^{21}$ carrying an integer topological charge identified with the baryon number,

$\mathcal{H}=-\frac{1}{96 \pi^{2}} \int d^{3} x \varepsilon_{i j k} \operatorname{tr}\left[L_{i} L_{j} L_{k}\right]$

where $\varepsilon_{i j k}$ is the antisymmetric Levi-Civita tensor $(i, j, k=x, y$ or $z)$ and $L_{i}=U^{\dagger} \partial_{i} U, U$ being an $S U(2)$ matrix describing the four meson fields $\left(\Phi_{0}, \Phi_{x}, \Phi_{y}, \Phi_{z}\right)$ on the 3-sphere $\left(\Phi_{0}^{2}+\Phi_{x}^{2}+\Phi_{y}^{2}+\Phi_{z}^{2}=1\right)$ : $U=\Phi_{0} \mathbb{1}+i \boldsymbol{\Phi} \cdot \boldsymbol{\sigma}$, where $\boldsymbol{\sigma}=\left(\sigma_{x}, \sigma_{y}, \sigma_{z}\right)$ is a vector composed of Pauli matrices. In the Skyrme's baryon, $\Phi_{0}$ depends on the radius $r$, varying from -1 (the south pole of the 3-sphere) at $r=0$ to +1 (the north pole) at infinity, and the vector $\Phi=\left(\Phi_{x}, \Phi_{y}, \Phi_{z}\right)$ is along the radius vector $\mathbf{r}$ (a hedgehog), which guarantees that the 3-sphere formed by the meson fields wraps once around the three-dimensional Euclidean space. This configuration was used as the initial state for numerical studies of 3D topological excitations in the uniform spiral state. 

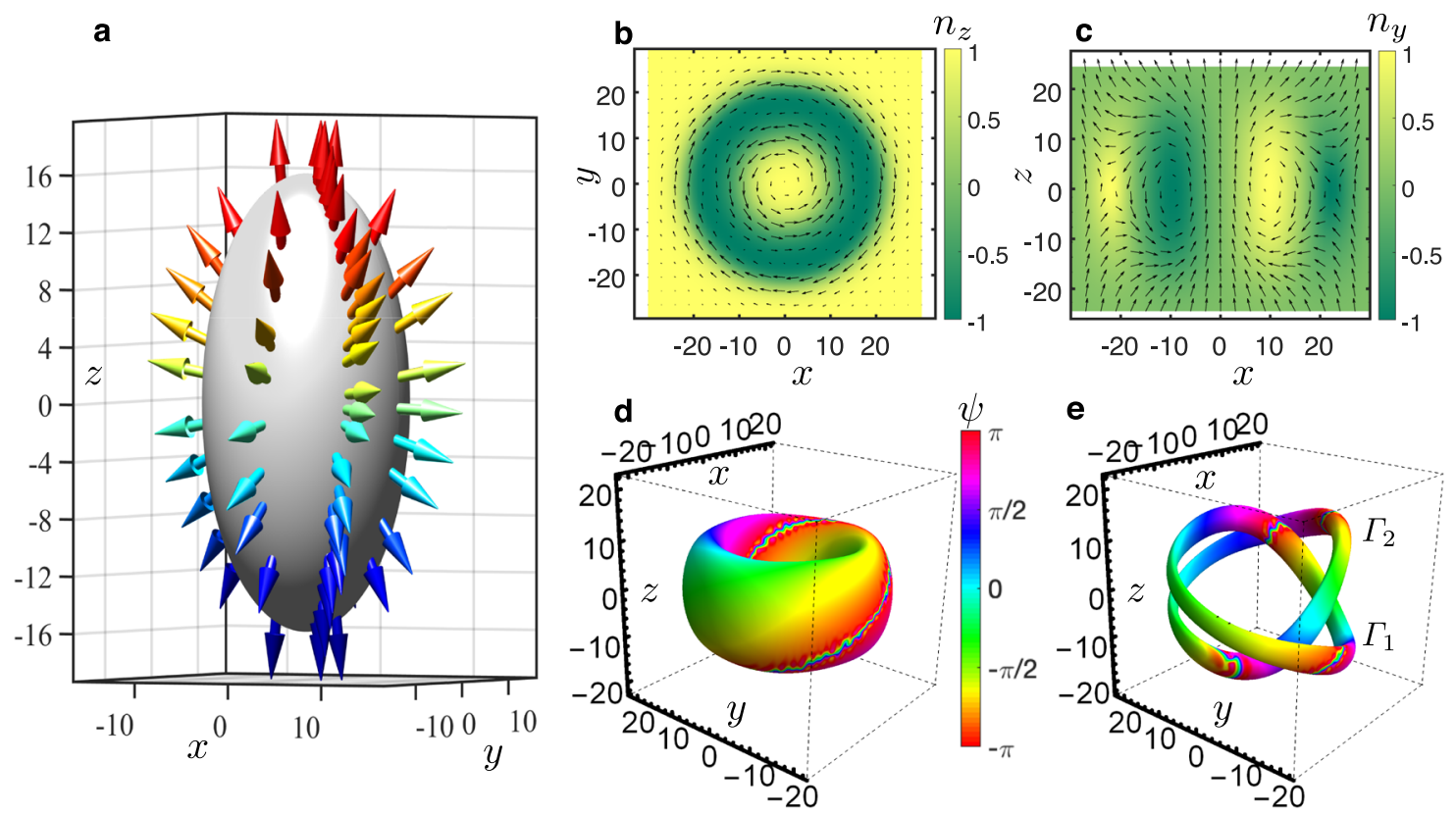

Fig. 7 Three-dimensional Skyrmion in a thin layer of Fe-langasite. a Arrows indicate the direction of $\Phi=\left(\Phi_{x}, \Phi_{y}, \Phi_{z}\right)$ at the $\Phi_{0}=0$ surface (gray ellipsoid). The corresponding $\mathbf{n}$-configuration in the $x y$ plane passing through $z=0$ (panel $\mathbf{b}$ ) and in the $x z$ plane passing through $y=0$ (panel c). d False color plot of the angle $\psi$ at the $n_{z}=-1 / 2$ surface. e Linking of two closed oriented paths, $\Gamma_{1,2}$, formed by the constant-n lines: $\mathbf{n}=(0, \sqrt{3} / 2,-1 / 2)^{\top}$, for $\Gamma_{1}$, and $\mathbf{n}=(\sqrt{3} / 2,0,-1 / 2)^{\top}$, for $\Gamma_{2}$. The numerical simulation was done for $J_{\perp}=J_{z}=1, \lambda=0.5, K_{1}=0, K_{2}=0.1, \mathbf{H}=0$ and an additional surface anisotropy with $K_{2}^{S}(z)=0.26 \cdot \exp \left(-\frac{\left(z_{0}-|z|\right)}{3.0}\right)$, where $z_{0}=25$ is half-width of the film.

The collapse of Skyrme's hedgehog is prevented by the terms of fourth order in spatial derivatives of $\Phi^{a}$. This stabilization mechanism is inefficient in Fe-langasite with relatively small fourth-order terms. 3D defects in chiral materials can be stabilized by DM interactions. The dependence of the 3D Skyrmion energy on the length scale $R$ (for a fixed shape) is given by $E(R)=a R-$ $b R^{2}+c R^{3}$, where the first term is the positive exchange energy, the second term is the negative energy resulting from the $\mathrm{LI}$, and the third term is the positive anisotropy energy counted from the energy of the uniform ground state $(a, b, c>0)$. The local minimum of $E(R)$ corresponding to a metastable Skyrmion appears for $b^{2}>3 a c$.

Our numerical simulations with periodic boundary conditions in all three directions show that $\lambda$ required to stabilize the 3D defect exceeds the critical value, above which the uniform spiral state becomes unstable towards additional periodic modulations, i.e., it transforms into the TS or DWA state in zero magnetic field. However, we have found a stable 3D Skyrmion in slabs with open boundary conditions along the $z$-direction, periodic boundary conditions along the $x$ and $y$ directions, and a surface anisotropy favoring $\mathbf{n}$ (anti)parallel to the $c$ axis, which suppresses the instability of the uniform state. This mechanism is similar to the stabilization of Hopfions in films of liquid crystals by boundary conditions ${ }^{48}$.

Figure 7a shows that the 3D Skyrmion is an axially symmetric hedgehog elongated along the $c$-axis. The gray surface is a surface of $\Phi_{0}=\cos \frac{\theta}{2} \cos \frac{\psi+\phi}{2}=0$ and the arrows show the direction of $\Phi=\left(\Phi_{x}, \Phi_{y}, \Phi_{z}\right)=\left(\sin \frac{\theta}{2} \sin \frac{\psi-\phi}{2}, \sin \frac{\theta}{2} \cos \frac{\psi-\phi}{2}, \cos \frac{\theta}{2} \sin \frac{\psi+\phi}{2}\right)$ at this surface. $\Phi_{0}=-1$ in the center of the 3D skyrmion and $\Phi_{0}=+1$ at the periphery so that the $\mathcal{H}=-1$ (see also Supplementary Note 5).

The $\mathbf{n}$-configuration in the $x y$ plane passing through the center of the defect is the 2D target skyrmion (Fig. 7b), whereas the $x z$ cut through the defect (see Fig. 7c) shows a doughnut shape. In fact, the $\mathbf{n}$-part of the 3D skyrmion is a Hopfion, similar to Hopfions in ferromagnets and liquid crystals ${ }^{49-53}$, and the 3D topological charge equals the Hopf number of the $\mathbf{n}$-texture written in terms of the vector potential $a_{i}=-D_{i} \psi=\mathbf{V}_{1} \cdot \partial_{i} \mathbf{V}_{2}$ and the corresponding magnetic field $\mathbf{b}=[\nabla \times \mathbf{a}]^{54,55}$ :

$\mathcal{H}=-\frac{1}{16 \pi^{2}} \int d^{3} x(\mathbf{a} \cdot \mathbf{b})$.

Figure $7 \mathrm{~d}$ shows the false-color plot of the angle $\psi$ at the $n_{z}=-1 / 2$ surface (a torus). The angle $\psi$ winds around the torus, which reflects the fact that the Hopf number is the linking number for constant-n loops ${ }^{54}$ (see Fig. 7e). The change of the angle $\psi$ along the loop, $\Delta \psi=-\phi \mathrm{d} \mathbf{x} \cdot \mathbf{a}=-4 \pi$. Importantly, the 3D Skyrmion is not merely a Hopfion, since the vector chirality $\mathbf{n}$ is only a part of the order parameter.

\section{DISCUSSION}

The importance of a larger order parameter space in triangular magnets with the $120^{\circ}$ ordering for critical phenomena and topological defects was noted already some time $\mathrm{ago}^{25}$. An additional ingredient discussed in this paper is the lack of inversion symmetry in the crystal lattice, which gives rise to DM interactions resulting in additional long-period modulations of the $120^{\circ}$ spin structure. The spin non-collinearity at the scale of one crystallographic unit cell gives rise to new magnetic phases and topological defects at a much larger length scale determined by the strength of the DM interactions.

We derived an effective model describing large-scale spin modulations in Fe-langasite, in particular the experimentally observed tilted spiral phase, and showed that the threedimensional order parameter space of this non-collinear antiferromagnet allows for complex spin states and unconventional topological magnetic defects, such as the coreless vortex tube and three-dimensional Skyrmion. The formal equivalence of the parameter spaces of antiferromagnets with a $120^{\circ}$ spin ordering and superfluid ${ }^{3} \mathrm{He}-\mathrm{A}$ calls for a study of magnetic analogs of the wealth of topological defects in the superfluid system ${ }^{2,3}$. 
Note, however, that ${ }^{3} \mathrm{He}$ and the Skyrme model do not allow for Lls stabilizing nanosized topological defects in the chiral Fe-langasite.

There are other non-collinear antiferromagnets with frustrated exchange interactions that can host unusual topological defects, such as manganese nitrides with the cubic inverse perovskite crystal structure showing a variety of non-collinear spin structures and a giant negative thermal expansion effect ${ }^{56-58}, \mathrm{~Pb}_{2} \mathrm{MnO}_{4}$ with a non-centrosymmetric tetragonal crystal lattice and a rare $90^{\circ}$ spin ordering ${ }^{59}$, multiferroic hexagonal manganites with a trimerized triangular spin-lattice and strongly coupled structural, ferroelectric and magnetic defects ${ }^{60-62}$, swedenborgites with alternating triangular and Kagome spin lattices, which similar to Fe-langasite are both frustrated and non-centrosymmetric ${ }^{40,63}$, and the conducting non-collinear antiferromagnets, $\mathrm{Mn}_{3} \mathrm{Ge}$ and $\mathrm{Mn}_{3} \mathrm{Sn}$, showing large anomalous Hall and Nernst effects and allowing for electric control of magnetic states $27-34,64$. The unconventional topological defects discussed in this paper can be a new avenue of research in AFM spintronics.

\section{METHODS}

\section{The effective description of the Heisenberg model}

The microscopic expression for the exchange energy with five exchange constants (see Fig. $1 \mathrm{~b}$ ) is written in terms of $\mathbf{V}_{1}$ and $\mathbf{V}_{2}$ and then, using Eq. (1), in terms of $\theta, \phi$ and $\psi$. Next, we use $\psi(\mathbf{r})=Q z+\psi(\mathbf{r})$ and average the exchange energy over short-period spin rotations. The resulting expression only depends on the slowly varying variables $\theta, \phi$ and $\psi$ and is expanded in powers of gradients of these three angles. Details can be found in Supplementary Note 1.

Lls for chiral antiferromagnets with a $120^{\circ}$ spin order, such as swedenborgaties and langasites, can be written in terms of the two vectors, $\mathbf{V}_{1}$ and $\mathbf{V}_{2}$, and their derivatives. They can be easily found using one-dimensional complex representations of $3_{z}$. To this end we introduce linear combinations of $\mathbf{V}_{1}=\left(X_{1}, Y_{1}, Z_{1}\right)$ and $\mathbf{V}_{2}=\left(X_{2}, Y_{2}, Z_{2}\right)$ :

$$
\begin{aligned}
R_{+} & =X_{1}+i X_{2}+i\left(Y_{1}+i Y_{2}\right)=\mathrm{e}^{\mathrm{i}(\phi-\psi)}(\cos \theta-1), \\
R_{-} & =X_{1}+i X_{2}-i\left(Y_{1}+i Y_{2}\right)=\mathrm{e}^{-\mathrm{i}(\phi+\psi)}(\cos \theta+1), \\
Z & =Z_{1}+i Z_{2}=-\sin \theta \mathrm{e}^{-\mathrm{i} \psi},
\end{aligned}
$$

and their complex conjugates denoted by $\bar{R}_{+}, \bar{R}_{-}$and $\bar{Z}$, respectively. These quantities transform in a simple way under the generators of the P321 group, $3_{z}$ and $2 y$ (see Table 1). These transformation rules follow directly from the symmetry properties of the order parameter:

$3_{z}: \phi \rightarrow \phi+\frac{2 \pi}{3}, \quad \Psi \rightarrow \Psi-\frac{2 \pi}{3}$,

$2_{y}: \phi \rightarrow-\phi, \quad \psi \rightarrow \pi-\psi$,

and $\theta$ being invariant under these transformations.

Using these transformation properties, one obtains five independent LIs favoring an additional modulation with an in-plane wave: $\operatorname{Im}\left(R_{+} \overleftrightarrow{\partial_{+}} R_{-}\right), \operatorname{Im}$ $\left(R_{+} \overleftrightarrow{\partial_{+}} \bar{R}_{-}\right), \operatorname{Im}\left(R_{+} \overleftrightarrow{\partial_{-}} \bar{Z}\right), \operatorname{Im}\left(R_{-} \overleftrightarrow{\partial_{+}} \bar{Z}\right)$, and $\operatorname{Im}\left(R_{-} \overleftrightarrow{\partial_{-}} Z\right)$, where $A \overleftrightarrow{\partial_{ \pm}} B=$ $A \partial_{i} B-B \partial_{i} A$ and $\partial_{+}=\partial_{x} \pm \partial \partial_{y}$. Two Lls vanish upon average over fast spin rotations ${ }^{39}$. Further details and the microscopic derivation of the LI from the DMI between nearest-neighbor triangles in the $a b$ plane can be found in Supplementary Note 2.

Table 1. Transformation properties of $R_{ \pm} Z$, and their complex conjugates (see Eq. (11)) under the generators of P321 group, $3_{z}$ and $z_{y}$ (here, $\omega=\mathrm{e}^{\mathrm{i} \frac{2 \pi}{3}}$ and $\bar{\omega}=\mathrm{e}^{-\mathrm{i} \frac{2 \pi}{3}}$ ).

\begin{tabular}{llc}
\hline & $3_{z}$ & $2_{y}$ \\
\hline$R_{+}$ & $\bar{\omega}$ & $-\bar{R}_{+}$ \\
$\bar{R}_{+}$ & $\omega$ & $-R_{+}$ \\
$R_{-}$ & 1 & $-\bar{R}_{-}$ \\
$\bar{R}_{-}$ & 1 & $-R_{-}$ \\
$Z$ & $\omega$ & $-\bar{Z}$ \\
$\bar{Z}$ & $\bar{\omega}$ & $-Z$ \\
\hline
\end{tabular}

\section{Numerical simulations}

We re-write the energy of the effective model Eq. (3) in terms of the unit vectors, $\mathbf{v}_{1}$ and $\mathbf{V}_{2}$ :

$$
\begin{aligned}
\mathcal{E}= & \frac{J_{z}}{2}\left[\left(\partial_{z} \mathbf{V}_{1}\right)^{2}+\left(\partial_{z} \mathbf{V}_{2}\right)^{2}\right]+\frac{J_{1}}{2} \sum_{\mu=x, y}\left[\left(\partial_{\mu} \mathbf{V}_{1}\right)^{2}+\left(\partial_{\mu} \mathbf{V}_{2}\right)^{2}\right] \\
& +K_{1}\left(1-n_{z}\right)+\frac{K_{2}}{2}\left(1-n_{z}^{2}\right)-\frac{x}{2}(\mathbf{H} \cdot \mathbf{n})^{2} \\
& -\lambda \sum_{i=1,2}\left[V_{i}^{x} \partial_{y} V_{i}^{z}-V_{i}^{y} \partial_{x} V_{i}^{z}\right]+J_{\text {ort }}\left(\mathbf{V}_{1} \cdot \mathbf{V}_{2}\right)^{2},
\end{aligned}
$$

where $\mathbf{n}=\mathbf{V}_{1} \times \mathbf{V}_{2}$, and $\mathbf{V}_{1}$ and $\mathbf{V}_{2}$ are slowly varying vectors with $\psi$ replaced by $\psi$. The term with a large $J_{\text {ort }}>0$ is added to ensure the orthogonality of $\mathbf{V}_{1}$ and $\mathbf{V}_{2}$. We then discretize Eq. (13) and minimize energy by solving two coupled Landau-Lifshitz-Gilbert equations for the unit vectors $\mathbf{V}_{1}$ and $\mathbf{V}_{2}$ with an artificially large Gilbert damping.

\section{DATA AVAILABILITY}

All data analyzed during the current study are available from the authors upon reasonable request.

Received: 18 August 2021; Accepted: 28 November 2021; Published online: 20 December 2021

\section{REFERENCES}

1. Mermin, N. D. The topological theory of defects in ordered media. Rev. Mod. Phys. 51, 591-648 (1979).

2. Vollhardt, D. \& Woelfle, P.The Superfluid Phases of Helium, Vol. 3 (CRC Press, 2003).

3. Volovik, G. Symmetry in Superfluid ${ }^{3} \mathrm{He}$. Helium Three 27-134 (Elsevier, 1990).

4. Derrick, G. H. Comments on nonlinear wave equations as models for elementary particles. J. Math. Phys. 5, 1252-1254 (1964).

5. Moriya, T. Anisotropic superexchange interaction and weak ferromagnetism. Phys. Rev. 120, 91-98 (1960).

6. Dzyaloshinskii, I. Theory of helicoidal structures in antiferromagnets I: Nonmetals. Sov. Phys. JETP 19, 960-971 (1964).

7. Bogdanov, A. N. \& Yablonskii, D. Thermodynamically stable "vortices" in magnetically ordered crystals. The mixed state of magnets. Sov. Phys. JETP 68, 101-103 (1989).

8. Mühlbauer, S. et al. Skyrmion lattice in a chiral magnet. Science 323, 915-919 (2009).

9. $\mathrm{Yu}, \mathrm{X}$. Z. et al. Real-space observation of a two-dimensional skyrmion crystal. Nature 465, 901-904 (2010).

10. Nagaosa, N. \& Tokura, Y. Topological properties and dynamics of magnetic skyrmions. Nat. Nanotechnol. 8, 899-911 (2013).

11. Fert, A., Cros, V. \& Sampaio, J. Skyrmions on the track. Nat. Nanotechnol. 8, 152-156 (2013).

12. Back, C. et al. The 2020 skyrmionics roadmap. J. Phys. D: Appl. Phys. 53, 363001 (2020).

13. Kurumaji, T. et al. Skyrmion lattice with a giant topological Hall effect in a frustrated triangular-lattice magnet. Science 365, 914-918 (2019).

14. Hirschberger, $M$. et al. Skyrmion phase and competing magnetic orders on a breathing kagomé lattice. Nat. Commun. 10, 5831 (2019).

15. Khanh, N. D. et al. Nanometric square skyrmion lattice in a centrosymmetric tetragonal magnet. Nat. Nanotechnol. 15, 444-449 (2020).

16. Okubo, T., Chung, S. \& Kawamura, H. Multiple- $q$ states and the skyrmion lattice of the triangular-lattice heisenberg antiferromagnet under magnetic fields. Phys. Rev. Lett. 108, 017206 (2012).

17. Leonov, A. O. \& Mostovoy, M. Multiply periodic states and isolated skyrmions in an anisotropic frustrated magnet. Nat. Commun. 6, 8275 (2015).

18. Gao, S. et al. Spiral spin-liquid and the emergence of a vortex-like state in $\mathrm{MnSc}_{2} \mathrm{~S}_{4}$. Nat. Phys. 13, 157-161 (2016).

19. Hayami, S., Ozawa, R. \& Motome, Y. Effective bilinear-biquadratic model for noncoplanar ordering in itinerant magnets. Phys. Rev. B 95, 224424 (2017).

20. Gao, S. et al. Fractional antiferromagnetic skyrmion lattice induced by anisotropic couplings. Nature 586, 37-41 (2020).

21. Skyrme, T. A unified field theory of mesons and baryons. Nucl. Phys. 31, 556-569 (1962).

22. Volovik, G. \& Mineev, V. Particle-like solitons in superfluid ${ }^{3}$ He phases. Zh. Eksp. Teor. Fiz 73, 767-773 (1977).

23. Shankar, R. Applications of topology to the study of ordered systems. J. Phys. $\mathbf{3 8}$, 1405-1412 (1977). 
24. Lee, W. et al. Synthetic electromagnetic knot in a three-dimensional skyrmion. Sci. Adv. 4, eaao3820 (2018).

25. Kawamura, H. \& Miyashita, S. Phase transition of the two-dimensional heisenberg antiferromagnet on the triangular lattice. J. Phys. Soc. Jpn. 53, 4138-4154 (1984).

26. Dombre, T. \& Read, N. Nonlinear $\sigma$ models for triangular quantum antiferromagnets. Phys. Rev. B 39, 6797-6801 (1989).

27. Chen, H., Niu, Q. \& MacDonald, A. Anomalous Hall effect arising from noncollinear antiferromagnetism. Phys. Rev. Lett. 112, 017205 (2014).

28. Kübler, J. \& Felser, C. Non-collinear antiferromagnets and the anomalous Hall effect. Europhys. Lett. 108, 67001 (2014).

29. Yang, H. et al. Topological Weyl semimetals in the chiral antiferromagnetic materials $\mathrm{Mn}_{3} \mathrm{Ge}$ and $\mathrm{Mn}_{3} \mathrm{Sn}$. New J. Phys. 19, 015008 (2017).

30. Kuroda, K. et al. Evidence for magnetic Weyl fermions in a correlated metal. Nat. Mater. 16, 1090-1095 (2017).

31. Li, B., Sandhoefner, S. \& Kovalev, A. A. Intrinsic spin Nernst effect of magnons in a noncollinear antiferromagnet. Phys. Rev. Res. 2, 013079 (2020).

32. Nakatsuji, S., Kiyohara, N. \& Higo, T. Large anomalous Hall effect in a non-collinear antiferromagnet at room temperature. Nature 527, 212-215 (2015).

33. Ikhlas, M. et al. Large anomalous Nernst effect at room temperature in a chiral antiferromagnet. Nat. Phys. 13, 1085-1090 (2017).

34. Tsai, $\mathrm{H}$. et al. Electrical manipulation of a topological antiferromagnetic state. Nature 580, 608-613 (2020)

35. Marty, K. et al. Single domain magnetic helicity and triangular chirality in structurally enantiopure $\mathrm{Ba}_{3} \mathrm{NbFe}_{3} \mathrm{Si}_{2} \mathrm{O}_{14}$. Phys. Rev. Lett. 101, 247201 (2008).

36. Stock, $C$. et al. Helical spin waves, magnetic order, and fluctuations in the langasite compound $\mathrm{Ba}_{3} \mathrm{NbFe}_{3} \mathrm{Si}_{2} \mathrm{O}_{14}$. Phys. Rev. B 83, 104426 (2011)

37. Loire, M. et al. Parity-broken chiral spin dynamics in $\mathrm{Ba}_{3} \mathrm{NbFe}_{3} \mathrm{Si}_{2} \mathrm{O}_{14}$. Phys. Rev. Lett. 106, 207201 (2011).

38. Zorko, A. et al. Role of antisymmetric exchange in selecting magnetic chirality in $\mathrm{Ba}_{3} \mathrm{NbFe}_{3} \mathrm{Si}_{2} \mathrm{O}_{14}$. Phys. Rev. Lett. 107, 257203 (2011).

39. Ramakrishnan, $M$. et al. Field-induced double spin spiral in a frustrated chiral magnet. npj Quantum Mater. 4, 60 (2019).

40. Reim, J. D. et al. Neutron diffraction study and theoretical analysis of the antiferromagnetic order and the diffuse scattering in the layered kagome system $\mathrm{CaBaCO}_{2} \mathrm{Fe}_{2} \mathrm{O}_{7}$. Phys. Rev. B 97, 144402 (2018).

41. Edmonds, A. R. Angular Momentum in Quantum Mechanics (Princeton University Press, 1960).

42. Chaix, L. et al. Helical bunching and symmetry lowering inducing multiferroicity in Fe langasites. Phys. Rev. B 93, 214419 (2016).

43. Bogdanov, A. N. \& Yablonskii, D. Contribution to the theory of inhomogeneous states of magnets in the region of magnetic-field-induced phase transitions. Mixed state of antiferromagnets. JETP 69, 142-146 (1989).

44. Mermin, N. D. \& Ho, T.-L. Circulation and angular momentum in the A phase of superfluid Helium-3. Phys. Rev. Lett. 36, 594-597 (1976).

45. Du, H., Ning, W., Tian, M. \& Zhang, Y. Magnetic vortex with skyrmionic core in a thin nanodisk of chiral magnets. Europhys. Lett. 101, 37001 (2013).

46. Leonov, A. O., Rößler, U. K. \& Mostovoy, M. Target-skyrmions and skyrmion clusters in nanowires of chiral magnets. EPJ Web Conf. 75, 05002 (2014).

47. Zheng, F. et al. Direct imaging of a zero-field target skyrmion and its polarity switch in a chiral magnetic nanodisk. Phys. Rev. Lett. 119, 197205 (2017).

48. Ackerman, P. J. \& Smalyukh, I. I. Static three-dimensional topological solitons in fluid chiral ferromagnets and colloids. Nat. Mater. 16, 426-432 (2016).

49. Cooper, N. R. Propagating magnetic vortex rings in ferromagnets. Phys. Rev. Lett. 82, 1554-1557 (1999).

50. Tai, J.-S. B. \& Smalyukh, I. I. Static Hopf solitons and knotted emergent fields in solid-state noncentrosymmetric magnetic nanostructures. Phys. Rev. Lett. 121, 187201 (2018).

51. Liu, Y., Lake, R. K. \& Zang, J. Binding a hopfion in a chiral magnet nanodisk. Phys. Rev. B 98, 174437 (2018)

52. Sutcliffe, P. Skyrmion knots in frustrated magnets. Phys. Rev. Lett. 118, 247203 (2017)

53. Rybakov, F. N. et al. Magnetic hopfions in solids. Preprint at arXiv http://arXiv.org/ abs/1904.00250 (2019).

54. Whitehead, J. H. C. An expression of Hopf's invariant as an integral. Proc. Natl. Acad. Sci. USA 33, 117-123 (1947).

55. Kosevich, A., Ivanov, B. \& Kovalev, A. Magnetic solitons. Phys. Rep. 194, 117-238 (1990).
56. likubo, S., Kodama, K., Takenaka, K., Takagi, H. \& Shamoto, S. Magnetovolume effect in $\mathrm{Mn}_{3} \mathrm{Cu}_{1-x} \mathrm{Ge}_{x} \mathrm{~N}$ related to the magnetic structure: Neutron powder diffraction measurements. Phys. Rev. B 77, 020409(R) (2008).

57. Kodama, K. et al. Gradual development of $\Gamma^{5 g}$ antiferromagnetic moment in the giant negative thermal expansion material $\mathrm{Mn}_{3} \mathrm{Cu}_{1-x} \mathrm{Ge}{ }_{x} \mathrm{~N}(x \sim 0.5)$. Phys. Rev. $B$ 81, 224419 (2010).

58. Mochizuki, M., Kobayashi, M., Okabe, R. \& Yamamoto, D. Spin model for nontrivial types of magnetic order in inverse-perovskite antiferromagnets. Phys. Rev. B 97, 060401(R) (2018).

59. Kimber, S. A. J. \& Attfield, J. P. Magnetic order in acentric $\mathrm{Pb}_{2} \mathrm{MnO}_{4}$. J. Mater Chem. 17, 4885-4888 (2007).

60. Lottermoser, T. et al. Magnetic phase control by an electric field. Nature 430 541-544 (2004).

61. Choi, T. et al. Insulating interlocked ferroelectric and structural antiphase domain walls in multiferroic $\mathrm{YMnO}_{3}$. Nat. Mater. 9, 253-258 (2010).

62. Artyukhin, S., Delaney, K. T., Spaldin, N. A. \& Mostovoy, M. Landau theory of topological defects in multiferroic hexagonal manganites. Nat. Mater. 13, 42-49 (2013).

63. Kocsis, V. et al. Magnetoelectric effect and magnetic phase diagram of a polar ferrimagnet $\mathrm{CaBaFe}_{4} \mathrm{O}_{7}$. Phys. Rev. B 93, 014444 (2016).

64. Liu, J. \& Balents, L. Anomalous Hall effect and topological defects in antiferromagnetic Weyl semimetals: $\mathrm{Mn}_{3} \mathrm{Sn} / \mathrm{Ge}$. Phys. Rev. Lett. 119, 087202 (2017).

\section{ACKNOWLEDGEMENTS}

We acknowledge Vrije FOM-programma 'Skyrmionics' and the Peregrine highperformance computing cluster.

\section{AUTHOR CONTRIBUTIONS}

E.B. and M.M. contributed equally to this work.

\section{COMPETING INTERESTS}

The authors declare no competing interests.

\section{ADDITIONAL INFORMATION}

Supplementary information The online version contains supplementary material available at https://doi.org/10.1038/s41535-021-00408-4.

Correspondence and requests for materials should be addressed to Evgenii Barts.

Reprints and permission information is available at http://www.nature.com/ reprints

Publisher's note Springer Nature remains neutral with regard to jurisdictional claims in published maps and institutional affiliations.

Open Access This article is licensed under a Creative Commons Attribution 4.0 International License, which permits use, sharing, adaptation, distribution and reproduction in any medium or format, as long as you give appropriate credit to the original author(s) and the source, provide a link to the Creative Commons license, and indicate if changes were made. The images or other third party material in this article are included in the article's Creative Commons license, unless indicated otherwise in a credit line to the material. If material is not included in the article's Creative Commons license and your intended use is not permitted by statutory regulation or exceeds the permitted use, you will need to obtain permission directly from the copyright holder. To view a copy of this license, visit http://creativecommons. org/licenses/by/4.0/.

(c) The Author(s) 2021, corrected publication 2022 